\title{
Surgery for secondary tricuspid regurgitation: Rings, bands, or sutures, does it matter?
}

\author{
Manuel J. Antunes, MD \\ From the University Hospital and Faculty of Medicine, Coimbra, Portugal. \\ Disclosures: Author has nothing to disclose with regard to commercial support. \\ Received for publication May 8, 2016; accepted for publication May 9, 2016. \\ Address for reprints: Manuel J. Antunes, MD, Cirurgia Cardiotorácica, Hospitais da Universidade de Coimbra, \\ 3000-075 Coimbra, Portugal (E-mail: antunes.cct.chuc@sapo.pt). \\ J Thorac Cardiovasc Surg 2016;152:416-7 \\ $0022-5223 / \$ 36.00$ \\ Copyright $(c) 2016$ by The American Association for Thoracic Surgery \\ http://dx.doi.org/10.1016/j.jtcvs.2016.05.032
}

In a work published in this edition of the Journal, Shinn and co-workers, ${ }^{1}$ from the Mayo Clinic, analyze the effectiveness of concomitant tricuspid valve (TV) repair in 479 patients undergoing operation for primary mitral valve disease from 1995 to 2010 . The majority of patients had degenerative disease $(54 \%)$, but rheumatic and ischemic cases were included. TV repair was performed using a flexible or rigid ring (54\%) and Kay or De Vega suture annuloplasty $(46 \%)$. Overall survival was $69 \%$ at 5 years and $44 \%$ at 10 years. Freedom from tricuspid regurgitation (TR) $3+$ or greater was $98 \%, 83 \%$, and $61 \%$ at dismissal, 4 years, and 8 years, respectively, and the method of tricuspid annuloplasty (and cause of the mitral valve disease) did not significantly influence the recurrence of TR.

This conclusion apparently is at odds with other recently published articles supporting the superiority of rings, but coincides with my beliefs, resulting from a large and significant experience with suture annuloplasty. ${ }^{2}$ In fact, there is still some controversy regarding the impact of suture versus prosthetic ring annuloplasty on long-term freedom from TR and reoperation in patients undergoing concomitant mitral valve surgery and TV repair. Although there are many studies recommending the use of ring annuloplasty over the De Vega suture annuloplasty, others have found no significant difference in outcome between the 2 methods, and there are a couple supporting the use of De Vega's suture annuloplasty over ring annuloplasty. ${ }^{3}$ A recently published meta-analysis by Parolari and colleagues ${ }^{4}$ concluded that ring annuloplasty is a protective factor for early mortality and long-term recurrence of TR compared with suture annuloplasty. Apparently, most agree in that there are no differences in survival.

The current article was presented at a major scientific meeting more than 2 years ago; thus, the data are somewhat outdated, but the number of patients is substantial. The use of each method of TV repair depended on the individual surgeon's preference, and the flexible rings were used mostly in the last 6 years of the study, whereas the relatively few rigid rings $(7 \%)$ were preferred initially. In addition, there is a newer generation of tricuspid rings, eventually more efficacious, that were not used in this study.

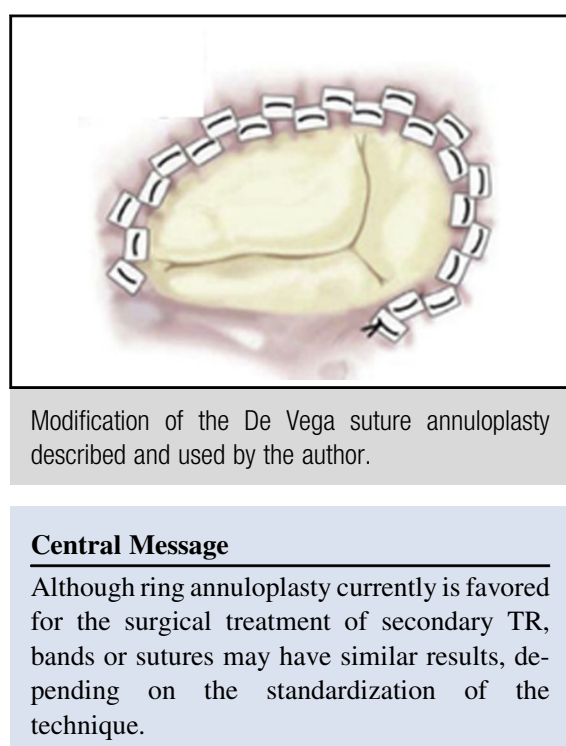

See Article page 406.
All of these may have introduced some inequalities in the analysis. Curiously, Shinn and co-workers ${ }^{1}$ mention this limitation when analyzing other previously published articles. Also, although this might not have been a primary point of investigation, the article does not give an indication of the authors' protocol, if there is one, for concomitant TV repair during surgery for mitral regurgitation. The rate of concomitant TV procedures also is not revealed. Finally, follow-up was short (mean, 2.7 years) and limited in numbers, because there was echocardiographic follow-up beyond 1 year postoperatively in only 209 of 422 eligible patients.

Clearly, there is still much to know about secondary TR and its treatment. ${ }^{5}$ Nonetheless, in my opinion and contrary to what happens with the mitral valve, where the final shape of the annulus is of paramount importance, in the TV, and in the absence of organic disease, it is the final size of the orifice that counts. And this makes tricuspid annuloplasty far less standardized, much more so with suture annuloplasty. This is also highlighted by the authors ${ }^{1}$ when describing their De Vega annuloplasty.

The Kay and De Vega procedures are 2 very different concepts. I am convinced that to work well the suture annuloplasty has to cover the circumference of the annulus to the same extent that rings do, that is, the whole of the anterior and posterior segments, and part of the septal. On the other hand, annular fibrosis after suture annuloplasty may, in fact, transform it into a flexible band. I have observed it with the 


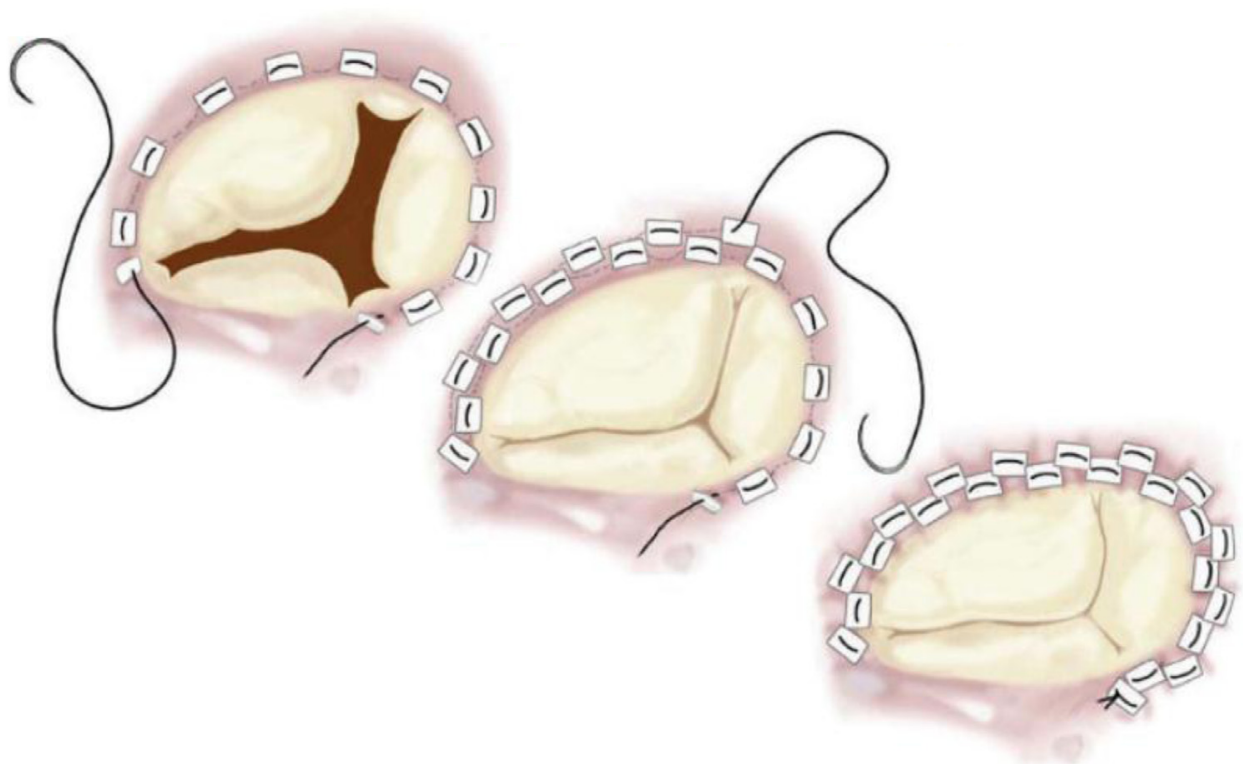

FIGURE 1. Modification of the De Vega tricuspid annuloplasty described in 1983. (Reproduced with permission from Antunes MJ, Barlow JB. Management of tricuspid valve regurgitation. Heart. 2007;93:271-6.)

modified technique I described in 1983, which uses interposed Teflon felt pledgets with every annular bite of the suture (Figure 1). One weakness of the suture annuloplasty is that it does not benefit from any commercial support.

\section{References}

1. Shinn SH, Dayan V, Schaff HV, Dearani J, Joyce LD, Lahr B, et al. Outcomes of ring versus suture annuloplasty for tricuspid valve repair in patients undergoing mitral valve surgery. J Thorac Cardiovasc Surg. 2016;152:406-15.e3.
2. Antunes MJ, Tornos Mas P, Rodríguez-Palomares JF. Secondary tricuspid valve regurgitation: a forgotten entity. Heart. 2015;101:1840-8.

3. Khorsandia M, Banerjeeb A, Singhb H, Srivastava AR. Is a tricuspid annuloplasty ring significantly better than a DeVega's annuloplasty stich when repairing severe tricuspid regurgitation? Interact Cardiovasc Thorac Surg. 2012;15: 129-35.

4. Parolari A, Barili F, Pilozzi A, Pacini D. Ring or suture annuloplasty for tricuspid regurgitation? A meta-analysis review. Ann Thorac Surg. 2014;98: 2255-63.

5. Dreyfrus G. Functional tricuspid regurgitation. A need to revise our understanding. J Am Coll Cardiol. 2015;65:2331-6. 